Bull. Korean Math. Soc. 50 (2013), No. 3, pp. 963-982

http://dx.doi.org/10.4134/BKMS.2013.50.3.963

\title{
ON THE GALERKIN-WAVELET METHOD FOR HIGHER ORDER DIFFERENTIAL EQUATIONS
}

\author{
NaOhiro Fukuda, Tamotu Kinoshita, and Takayuki Kubo
}

\begin{abstract}
The Galerkin method has been developed mainly for 2nd order differential equations. To get numerical solutions, there are some choices of Riesz bases for the approximation subspace $V_{j} \subset L^{2}$. In this paper we shall propose a uniform approach to find suitable Riesz bases for higher order differential equations. Especially for the beam equation (4-th order equation), we also report numerical results.
\end{abstract}

\section{Introduction}

\subsection{The Galerkin method}

The Galerkin method is a numerical method for converting a continuous operator problem to a discrete problem (see, [10, 13, 15], etc.). With respect to the bilinear form, the approximation error between the solution $u$ of the differential equation and the solution $u_{j}$ of the Galerkin equation is orthogonal to the approximation subspace $V_{j} \subset L^{2}$, where $j$ is a fixed dilation parameter. For instance, let us consider the $2 m$-th order differential equation $(-1)^{m} \frac{d^{2 m}}{d x^{2 m}} u+u=f$ and its Galerkin equation $a\left(u_{j}, \varphi_{j, k}\right)=\left\langle f, \varphi_{j, k}\right\rangle_{L^{2}}$, where

$$
a(g, \phi)=\langle g, \phi\rangle_{L^{2}}+\left\langle\frac{d^{m}}{d x^{m}} g, \frac{d^{m}}{d x^{m}} \phi\right\rangle_{L^{2}} .
$$

Then the error $e_{j}:=u-u_{j}$ satisfies by integration by parts

$$
a\left(e_{j}, \varphi_{j, k}\right)=\left\langle\left((-1)^{m} \frac{d^{2 m}}{d x^{2 m}}+1\right) u, \varphi_{j, k}\right\rangle_{L^{2}}-a\left(u_{j}, \varphi_{j, k}\right)=0
$$

for all $\varphi_{j, k}(k \in \mathbb{Z})$, where $k$ is the translation parameter to span the whole $V_{j}$. The subspace $V_{j}$ on which $u$ is projected, characterizes the visual shape of $u_{j}$. However, it is not always concerned with the sharpness of the approximation, because the residual error $e_{j}$ is often estimated by the base which is not uniquely

Received April 19, 2012; Revised September 26, 2012.

2010 Mathematics Subject Classification. 65N30, 65L60, 80M10.

Key words and phrases. Galerkin-wavelet method, Riesz basis, higher order differential equation. 
determined for the subspace $V_{j}$. So, the choices of the subspace and its base are up to us.

Generally, the compactly-supported bases are preferable to get numerical solutions of differential equations. In particular, the Galerkin method with piecewise polynomials called Finite element method (FEM). These compactlysupported bases enable us to localize a few elements in the whole interval, but are not orthogonal in translation. The Galerkin-wavelet method can be dealt with the (sufficiently smooth) Daubechies scaling function (or wavelet) which is not only orthogonal but also compactly-supported (see $[1,5]$ ). To get a better approximation, there are two ways: by increasing the degree of the scaling function (or wavelet) and the level $j$. In general, the stiffness matrix with orthogonal bases are full. To consider higher order differential equations, orthogonal bases require many computations of stiffness matrix. In this paper, our aim is to find suitable (non orthogonal) Riesz bases for higher order differential equations in the sense that stiffness matrices are more sparse.

\subsection{Uniform approach to find suitable bases}

According to differential equations, we expect certain smoothness (at least Lipschitz continuity) for the subspace. Let us put the B-splines of orders 1 and 2 as follows:

$$
N_{1}(x)=\left\{\begin{array}{ll}
1 & \text { if } 0 \leq x \leq 1, \\
0 & \text { otherwise, }
\end{array} \quad N_{2}(x)= \begin{cases}x & \text { if } 0 \leq x \leq 1 \\
2-x & \text { if } 1 \leq x \leq 2 \\
0 & \text { otherwise }\end{cases}\right.
$$

$N_{1}(x)$ is called the Haar scaling function. $\left\{N_{2}(x-k): k \in \mathbb{Z}\right\}$ which is a Riesz basis for the space $V_{0}$ of piecewise linear continuous functions on the intervals $[k, k+1]$ for all $k \in \mathbb{Z}$, is used in the standard FEM. We remark that the Franklin scaling function and the Strömberg scaling function can be also orthogonal bases for $V_{0}$ (see $[7,11,14]$ ). The Lipschitz continuity of functions in the subspace comes from the property of these bases. Therefore, our task is to determine a base scaling function rather than a subspace.

From the point of view of the study of differential equations, the coefficient of the highest order derivative has much more influence on the behavior of the solution. After the translation of the continuous problem into the discrete one, if the matrix corresponding to the principal part becomes simpler, the approximate solution will be more stable as an appropriate numerical treatment. In this section we shall give a uniform approach to find suitable bases such that the matrix corresponding to the principal part has just a form of 3-point formula or more generally, $(2 m+1)$-point formula.

At first, for the simplicity, let us consider the 2 nd order equation $-\frac{d^{2}}{d x^{2}} u+u=$ $f$ and $V_{0}$, i.e., $j=0$. We are concerned with the following matrix coming from the principal part:

$$
a_{k, \ell}:=-\left\langle\frac{d}{d x} \varphi_{0, k}, \frac{d}{d x} \varphi_{0, \ell}\right\rangle_{L^{2}}\left(=\left\langle\frac{d^{2}}{d x^{2}} \varphi_{0, k}, \varphi_{0, \ell}\right\rangle_{L^{2}} \text { if } \varphi \in C^{2}\right) .
$$


Since $\varphi_{0, k}(x)=\varphi(x-k)$, by Parseval's theorem we see that

$$
\left\langle\frac{d}{d x} \varphi_{0, k}, \frac{d}{d x} \varphi_{0, \ell}\right\rangle_{L^{2}}=\frac{1}{2 \pi}\left\langle i \xi e^{-i k \xi} \hat{\varphi}, i \xi e^{-i \ell \xi} \hat{\varphi}\right\rangle_{L^{2}}=\mathcal{F}^{-1}\left[|\xi \hat{\varphi}(\xi)|^{2}\right](\ell-k) .
$$

On the other hand, in order to get 3-point formula for 2 nd order derivative, we need the tridiagonal matrix

$$
\left\{a_{k, \ell}\right\}_{1 \leq k, \ell \leq N}=\left(\begin{array}{ccccccc}
-2 & 1 & 0 & \cdots & \cdots & \cdots & 0 \\
1 & -2 & 1 & 0 & \cdots & \cdots & 0 \\
0 & 1 & -2 & 1 & 0 & \cdots & 0 \\
\vdots & \ddots & \ddots & \ddots & \ddots & \ddots & \vdots \\
\vdots & & \ddots & \ddots & \ddots & \ddots & 0 \\
0 & \ldots & \cdots & 0 & 1 & -2 & 1 \\
0 & \cdots & \cdots & 0 & 0 & 1 & -2
\end{array}\right)
$$

where $N$ depends on the interval in which $-\frac{d^{2}}{d x^{2}} u+u=f$ is considered. Thus, $\varphi$ must satisfy the condition

$$
\mathcal{F}^{-1}\left[|\xi \hat{\varphi}(\xi)|^{2}\right](\ell-k)=\left\{\begin{array}{cl}
2 & \text { if } k=\ell \\
-1 & \text { if } k=\ell \pm 1 \\
0 & \text { otherwise }
\end{array}\right.
$$

It would not be easy to find $\varphi$ from (1.2). Therefore, we shall try to change the condition (1.2).

Further computations yield

$$
\begin{aligned}
\mathcal{F}^{-1}\left[|\xi \hat{\varphi}(\xi)|^{2}\right](\ell-k) & =\frac{1}{2 \pi} \sum_{q \in \mathbb{Z}} \int_{2 q \pi}^{2(q+1) \pi} e^{i(\ell-k) \xi}|\xi \hat{\varphi}(\xi)|^{2} d \xi \\
& =\frac{1}{2 \pi} \sum_{q \in \mathbb{Z}} \int_{0}^{2 \pi} e^{i(\ell-k) \xi}|(\xi+2 q \pi) \hat{\varphi}(\xi+2 q \pi)|^{2} d \xi \\
& =\frac{1}{2 \pi} \int_{0}^{2 \pi} e^{i(\ell-k) \xi} \sum_{q \in \mathbb{Z}}|(\xi+2 q \pi) \hat{\varphi}(\xi+2 q \pi)|^{2} d \xi
\end{aligned}
$$

Hence, we find that (1.2) is equivalent to

$$
\sum_{q \in \mathbb{Z}}|(\xi+2 q \pi) \hat{\varphi}(\xi+2 q \pi)|^{2} \equiv-e^{i \xi}+2 e^{i 0 \xi}-e^{-i \xi}\left(=4 \sin ^{2} \frac{\xi}{2}\right)
$$

for almost everywhere $\xi \in \mathbb{R}$. Denoting the sinc function by $\operatorname{sinc} \xi=\frac{\sin \xi}{\xi}$, we see that the Haar scaling function $N_{1}(x)$ satisfies $\hat{N}_{1}(\xi)=e^{-i \xi / 2} \operatorname{sinc} \frac{\xi}{2}$. We shall define $\Phi(x)$ by

$$
\hat{\Phi}(\xi)=\frac{\hat{\varphi}(\xi)}{\hat{N}_{1}(\xi)}
$$


Then we also get

$$
\sum_{q \in \mathbb{Z}}|\hat{\Phi}(\xi+2 q \pi)|^{2}=\sum_{q \in \mathbb{Z}} \frac{|(\xi+2 q \pi) \hat{\varphi}(\xi+2 q \pi)|^{2}}{4 \sin ^{2} \frac{\xi+2 q \pi}{2}}=\frac{\sum_{q \in \mathbb{Z}}|(\xi+2 q \pi) \hat{\varphi}(\xi+2 q \pi)|^{2}}{-e^{i \xi}+2 e^{i 0 \xi}-e^{-i \xi}},
$$

here we used

$$
4 \sin ^{2} \frac{\xi+2 q \pi}{2}=4 \sin ^{2} \frac{\xi}{2}=-e^{i \xi}+2 e^{i 0 \xi}-e^{-i \xi}
$$

This means that

$$
\sum_{q \in \mathbb{Z}}|\hat{\Phi}(\xi+2 q \pi)|^{2} \equiv 1 \quad \text { a.e. } \xi \in \mathbb{R} .
$$

So, the condition (1.2) has been reduced to the conditions (1.3) and (1.4). Now we can easily find $\varphi$ from (1.3) and (1.4), because the identity (1.4) is well-known as the orthonormal condition. The definition (1.3) yields

$$
\varphi(x)=\mathcal{F}^{-1}\left[\hat{N}_{1}(\xi) \hat{\Phi}(\xi)\right](x)=N_{1} * \Phi(x)\left(=\int_{x-1}^{x} \Phi(y) d y\right) .
$$

The new function $\varphi$ is the elevation of $\Phi$ with $N_{1}$. Therefore $N_{1}$ is also called the elevator (see $[16,17]$ ). More generally, let us represent the elevator by $\mathcal{E}$ and define

$$
\varphi(x)=\mathcal{E} * \Phi(x) .
$$

Remark 1.1. The most typical example is the case when the elevator $\mathcal{E}(x)$ is $N_{1}(x)$ and $\Phi(x)$ is the Haar scaling function, i.e., $\mathcal{E}(x)=\Phi(x)=N_{1}(x)$. By (1.5) we obtain

$$
\varphi(x)=N_{1} * N_{1}(x)=N_{2}(x) .
$$

This case just coincides with the standard FEM. Choosing other scaling functions for $\Phi(x)$, we can obtain various types of bases.

\subsection{Definition of elevator}

We shall derive some properties for the case when the elevator $\mathcal{E}(x)$ is $N_{1}(x)$. By Taylor expansion we see that for $v \in C^{4}$

$$
\sum_{\nu=-1}^{1} a_{k, k+\nu} v(x+\nu h)=h^{2} \frac{d^{2}}{d x^{2}} v(x)+O\left(h^{4}\right) \text { for all } k \in \mathbb{Z} .
$$

Moreover, we assume that

$$
\hat{\Phi}(0)=1,
$$

which allows scaling functions, but excludes wavelet functions. Hence, by (1.5) it follows that

$$
\sum_{k \in \mathbb{Z}} \varphi_{j, k}(x)=\sum_{k \in \mathbb{Z}} \varphi\left(2^{j} x-k\right)=\sum_{k \in \mathbb{Z}} \int_{2^{j} x-k-1}^{2^{j} x-k} \Phi(y) d y
$$




$$
=\int_{-\infty}^{\infty} \Phi(y) d y=\hat{\Phi}(0)=1 .
$$

This is just the partition of unity. Let us put $h=2^{-j}$ and

$$
w_{j}(x)=\sum_{\ell \in \mathbb{Z}} w_{j, \ell} \varphi_{j, \ell}(x) .
$$

If $w_{j}$ is sufficiently smooth and $\varphi$ has compact support (or decays sufficiently fast), by (1.9) we have for $k \in \mathbb{Z}$

$$
w_{j}(k h)=\sum_{\ell \in \mathbb{Z}} w_{j, \ell} \varphi_{j, \ell}(k h) \sim w_{j, k} \sum_{\ell \in \mathbb{Z}} \varphi_{j, \ell}(k h)=w_{j, k} .
$$

Indeed, it holds that $w_{j}(k h)=w_{j, k}$ in the standard FEM.

Meanwhile we also get the following identity:

$$
\sum_{q \in \mathbb{Z}}|\hat{\varphi}(\xi+2 q \pi)|^{2}=\sum_{\nu \in \mathbb{Z}} c_{k, k+\nu} e^{i \nu \xi},
$$

where $c_{k, \ell}:=\left\langle\varphi_{0, k}, \varphi_{0, \ell}\right\rangle_{L^{2}}$. In particular, taking $\xi=0$, by (1.3) and (1.8) we find that for all $k \in \mathbb{Z}$

$$
\sum_{\nu \in \mathbb{Z}} c_{k, k+\nu}=\sum_{q \in \mathbb{Z}}|\hat{\varphi}(2 q \pi)|^{2}=\sum_{q \in \mathbb{Z}}\left|\hat{N}_{1}(2 q \pi) \hat{\Phi}(2 q \pi)\right|^{2}=|\hat{\Phi}(0)|^{2}=1,
$$

here we used $\hat{\Phi}(2 q \pi)=0$ if $q \neq 0$, since $\sum_{q \neq 0}|\hat{\Phi}(2 q \pi)|^{2}-|\hat{\Phi}(0)|^{2}=0$ by (1.8) and (1.4) with $\xi=0$. Noting that $c_{k, k+\nu}=c_{k, k-\nu}$, by Taylor expansion we see that for $v \in C_{0}^{2}$

$$
\sum_{\nu \in \mathbb{Z}} c_{k, k+\nu} v(x+\nu h)=v(x)+O\left(h^{2}\right) \text { for all } k \in \mathbb{Z} .
$$

In our construction, to get the approximate solution $u_{j}(x)=\sum_{\ell \in \mathbb{Z}} u_{j, \ell} \varphi_{j, \ell}(x)$ in the interval $(0,1)$ for the equation $-\frac{d^{2}}{d x^{2}} u+u=f$, we solve the following system corresponding to the Galerkin equation:

$$
\begin{aligned}
& {\left[-\left\{a_{k, \ell} h^{-2}\right\}_{1 \leq k, \ell \leq N}+\left\{c_{k, \ell} h\right\}_{1 \leq k, \ell \leq N}\right]{ }^{t}\left\{u_{j, \ell}\right\}_{1 \leq \ell \leq N} } \\
= & \left\{c_{k, \ell}\right\}_{1 \leq k, \ell \leq N}{ }^{t}\left\{f_{j, \ell}\right\}_{1 \leq \ell \leq N} .
\end{aligned}
$$

By (1.10) this can be regarded as

$$
\begin{aligned}
& {\left[-\left\{a_{k, \ell} h^{-2}\right\}_{1 \leq k, \ell \leq N}+\left\{c_{k, \ell} h\right\}_{1 \leq k, \ell \leq N}\right]^{t}\left\{u_{j}(\ell h)\right\}_{1 \leq \ell \leq N} } \\
= & \left\{c_{k, \ell}\right\}_{1 \leq k, \ell \leq N}{ }^{t}\left\{f_{j}(\ell h)\right\}_{1 \leq \ell \leq N} .
\end{aligned}
$$

Paying attention to each row, by (1.7) and (1.11) we find that

$-\sum_{1 \leq \ell \leq 2^{j}} a_{k, \ell} h^{-2} u_{j}(\ell h)=-\sum_{\nu} a_{k, k+\nu} h^{-2} u_{j}(k h+\nu h)=-\frac{d^{2}}{d x^{2}} u_{j}(k h)+O\left(h^{2}\right)$, 


$$
\begin{aligned}
& \sum_{1 \leq \ell \leq 2^{j}} c_{k, \ell} u_{j}(\ell h)=\sum_{\nu} c_{k, k+\nu} u_{j}(k h+\nu h)=u_{j}(k h)+O\left(h^{2}\right), \\
& \sum_{1 \leq \ell \leq 2^{j}} c_{k, \ell} f_{j}(\ell h)=\sum_{\nu} c_{k, k+\nu} f_{j}(k h+\nu h)=f_{j}(k h)+O\left(h^{2}\right) .
\end{aligned}
$$

These give the numerical difference equation of the original differential equation $-\frac{d^{2}}{d x^{2}} u+u=f$ at the point $x=k h$. The accuracy of (1.10) depends on the case of application. We remark that (1.7) and (1.11) play an important role to guarantee the accuracy.

From the above observations for $\mathcal{E}=N_{1}$, we shall propose the following conditions to characterize qualitative elevators for the Galerkin method:

Definition 1.1. Let $\Phi$ be a scaling function such that $\hat{\Phi}(0)=1$ and $\hat{\Phi}(\xi) \neq 0$ for $-\pi \leq \xi \leq \pi$. Put $c_{k, \ell}:=\left\langle\varphi_{0, k}, \varphi_{0, \ell}\right\rangle_{L^{2}}$ and $a_{k, \ell}:=-\left\langle\frac{d}{d x} \varphi_{0, k}, \frac{d}{d x} \varphi_{0, \ell}\right\rangle_{L^{2}}$ for $\varphi(x)=\mathcal{E} * \Phi(x)$. The elevator $\mathcal{E}$ for the Galerkin method is a function satisfying

(i) $\hat{\mathcal{E}}(\xi) \neq 0$ for $-\pi \leq \xi \leq \pi$, in particular, $\hat{\mathcal{E}}(0)=1$.

(ii) It holds that for $v \in C_{0}^{4}$

$$
\begin{aligned}
& \sum_{\nu \in \mathbb{Z}} c_{k, k+\nu} v(x+\nu h)=v(x)+O\left(h^{2}\right), \\
& \sum_{\nu \in \mathbb{Z}} a_{k, k+\nu} v(x+\nu h)=h^{2} \frac{d^{2}}{d x^{2}} v(x)+O\left(h^{4}\right) .
\end{aligned}
$$

(iii) There exists a $2 \pi$-periodic function $m_{\mathcal{E}}(\xi)$ such that $\hat{\mathcal{E}}(2 \xi)=m_{\mathcal{E}}(\xi) \hat{\mathcal{E}}(\xi)$.

It is known that the exact frame is equivalent to the Riesz basis. The condition for the Riesz basis is given by

$$
A \leq \sum_{q \in \mathbb{Z}}|\hat{\varphi}(\xi+2 q \pi)|^{2} \leq B
$$

for $0<A \leq B<\infty$ (see [2]). If $\hat{\Phi}(\xi) \neq 0$ for $-\pi \leq \xi \leq \pi$, by (i) we note that

$$
\begin{aligned}
\sum_{q \in \mathbb{Z}}|\hat{\varphi}(\xi+2 q \pi)|^{2} & =\sum_{q \in \mathbb{Z}}|\hat{\mathcal{E}}(\xi+2 q \pi) \hat{\Phi}(\xi+2 q \pi)|^{2} \\
& \geq|\hat{\mathcal{E}}(\xi-2 n \pi) \hat{\Phi}(\xi-2 n \pi)|^{2} \\
& \geq{ }^{\exists} A>0
\end{aligned}
$$

for $2 n \pi-\pi \leq \xi \leq 2 n \pi+\pi(n \in \mathbb{Z})$, that is, $\xi \in \mathbb{R}$. Rewriting $\varphi(x)=N_{1} * \Phi^{\sharp}(x)$ with $\hat{\Phi}^{\sharp}(\xi)=\frac{\hat{\mathcal{E}}(\xi) \hat{\Phi}(\xi)}{\hat{N}_{1}(\xi)}$, from (i) we can expect that the properties corresponding to $(1.9),(1.10)$ and $(1.11)$ still hold, since $\hat{\Phi}^{\sharp}(0)=1$. In fact, we may omit $\sum_{\nu \in \mathbb{Z}} c_{k, k+\nu} v(x+\nu h)=v(x)+O\left(h^{2}\right)$ in (ii).

Replacing the definition $\varphi_{j, k}(x)=\varphi\left(2^{j} x-k\right)$ by $\varphi_{j, k}(x)=2^{j / 2} \varphi\left(2^{j} x-\right.$ $k$ ), we could also get wavelet expansions. Thanks to the condition (iii) we obtain a semi-orthogonal wavelet $\hat{\psi}(\xi)=e^{i \xi / 2} \overline{m(\xi / 2+\pi)} \hat{\varphi}(\xi / 2)$, where $m(\xi)=$ 


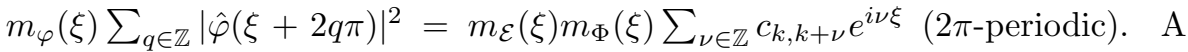
biorthogonal wavelet for the elevated $\varphi$ can be also considered (see [6]). By (1.7) we have already found the elevator $\mathcal{E}$ which allows the finite sum of $a_{k, k+\nu} v(x+$ $\nu h$ ) in (ii) and improves the standard FEM with $\varphi=N_{2}$ in $\S 4$.

\section{Riesz basis of Daubechies type}

\subsection{3-point formula for 2 nd order derivative}

To get compactly-supported and also more smooth base than $N_{2}$, we may choose the Daubechies scaling function of order $p$ for $\Phi \equiv \Phi_{p}^{D}$ satisfying (1.8). Then by (1.5) we have

$$
\varphi_{p}^{D}(x)=N_{1} * \Phi_{p}^{D}(x)
$$

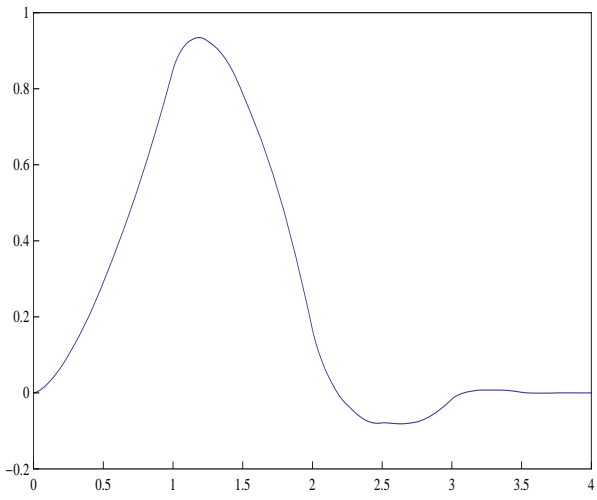

(a) $\varphi_{2}^{D}\left(\operatorname{supp} \varphi_{2}^{D} \subset[0,4]\right)$

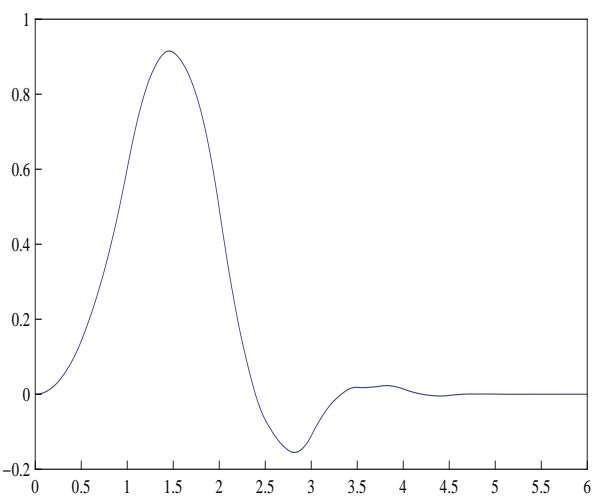

(b) $\varphi_{3}^{D}\left(\operatorname{supp} \varphi_{3}^{D} \subset[0,6]\right)$

FiguRE 1. Graphs of $\varphi_{2}^{D}$ and $\varphi_{3}^{D}$. 
The basis $\left\{\varphi_{p}^{D}(x-k): k \in \mathbb{Z}\right\}$ had been derived by [16] and [17]. Their approach is motivated from the observation that the integration of the Haar wavelet becomes $N_{2}$. Therefore, the pseudoframe was firstly considered by the integration of the Daubechies wavelet, and secondly it was arranged for the efficiency of the computation and arrived at $\varphi_{p}^{D}$ (see also [12]).

In order to solve numerically the equation $-\frac{d^{2}}{d x^{2}} u+u=f$ with some base $\{\varphi(x-k): k \in \mathbb{Z}\}$, we need to know the matrices $\left\{c_{k, \ell}\right\}_{1 \leq k, \ell \leq N}$ and $\left\{a_{k, \ell}\right\}_{1 \leq k, \ell \leq N}$. If one considers the orthogonal Daubechies scaling function, it holds that the matrix $\left\{c_{k, \ell}\right\}_{1 \leq k, \ell \leq N}=I$. On the other hand, the matrix $\left\{a_{k, \ell}\right\}_{1 \leq k, \ell \leq N}$ for the Daubechies scaling function is well studied in [1]. For all the bases constructed by the approach in $\S 1.2$, the matrix $\left\{a_{k, \ell}\right\}_{1 \leq k, \ell \leq N}$ is just (1.1).

Remark 2.1. It would be preferable that bases are at least $C^{1}$ or Lipschitz continuous as $N_{2}$ in order that the weak form $-\left\langle\frac{d}{d x} \varphi_{0, k}, \frac{d}{d x} \varphi_{0, \ell}\right\rangle_{L^{2}}$ of $\left\langle\frac{d^{2}}{d x^{2}} \varphi_{0, k}\right.$, $\left.\varphi_{0, \ell}\right\rangle_{L^{2}}$ has a meaning. Especially for $p=2$, the Daubechies scaling function $\Phi_{2}^{D} \in C^{0.55}$ fails to satisfy the differentiability, but gives $\varphi_{2}^{D} \in C^{1.55}$.

We shall also compute the exact value of $c_{k, \ell}$ for $\varphi_{2}^{D}(x)$. Putting $\hat{\phi}(\xi)=$ $\left|\hat{\varphi}_{2}^{D}(\xi)\right|^{2}$, by Parseval's theorem we have

$$
c_{k, \ell}=\left\langle\varphi_{2}^{D}(x-k), \varphi_{2}^{D}(x-\ell)\right\rangle_{L^{2}}=\phi(\ell-k) .
$$

By (2.1) it holds that

$$
\hat{\phi}(\xi)=\operatorname{sinc}^{2} \frac{\xi}{2} \hat{\Phi}_{2}^{D}(\xi)^{2}=\prod_{j=1}^{\infty} \cos ^{2}\left(\frac{\xi}{2^{j+1}}\right)\left|m_{2}^{D}\left(\frac{\xi}{2^{j}}\right)\right|^{2} \equiv \prod_{j=1}^{\infty} \tilde{m}\left(\frac{\xi}{2^{j}}\right),
$$

where $m_{2}^{D}$ is the Daubechies low pass filter. Since $m_{2}^{D}(\xi)=\sum_{k=0}^{3} \eta_{k} e^{-i k \xi}$ with $\left\{\eta_{0}, \eta_{1}, \eta_{2}, \eta_{3}\right\}=\left\{\frac{1+\sqrt{3}}{8}, \frac{3+\sqrt{3}}{8}, \frac{3-\sqrt{3}}{8}, \frac{1-\sqrt{3}}{8}\right\}$ and $\cos ^{2} \xi=\frac{e^{2 i \xi}+2+e^{-2 i \xi}}{4}$, we find that $\tilde{m}(\xi)=\sum_{k=-4}^{4} \mu_{k} e^{-i k \xi}$ and its coefficients are given by

$$
\left\{\mu_{0}, \mu_{ \pm 1}, \mu_{ \pm 2}, \mu_{ \pm 3}, \mu_{ \pm 4}\right\}=\left\{\frac{25}{64}, \frac{17}{64}, \frac{1}{16},-\frac{1}{64},-\frac{1}{128}\right\} .
$$

The function $\phi$ satisfies

$$
\phi(x)=2 \sum_{k=-4}^{4} \mu_{k} \phi(2 x-k)
$$

and $\operatorname{supp} \phi \subset[-4,4]$ since $\operatorname{supp} \varphi_{2}^{D} \subset[0,4]$ and $\phi(x)=\int_{\mathbb{R}} \varphi_{2}^{D}(t+x) \overline{\varphi_{2}^{D}(t)} d t$. Hence we have

$$
M^{t}\{\phi(k)\}_{-3 \leq k \leq 3}=\mathbf{0},
$$


where

$$
M=\left(\begin{array}{ccccccc}
1-2 \mu_{-3} & -2 \mu_{-4} & 0 & 0 & 0 & 0 & 0 \\
-2 \mu_{-1} & 1-2 \mu_{-2} & -2 \mu_{-3} & -2 \mu_{-4} & 0 & 0 & 0 \\
-2 \mu_{1} & -2 \mu_{0} & 1-2 \mu_{-1} & -2 \mu_{-2} & -2 \mu_{-3} & -2 \mu_{-4} & 0 \\
-2 \mu_{3} & -2 \mu_{2} & -2 \mu_{1} & 1-2 \mu_{0} & -2 \mu_{-1} & -2 \mu_{-2} & -2 \mu_{-3} \\
0 & -2 \mu_{4} & -2 \mu_{3} & -2 \mu_{2} & 1-2 \mu_{1} & -2 \mu_{0} & -2 \mu_{-1} \\
0 & 0 & 0 & -2 \mu_{4} & -2 \mu_{3} & 1-2 \mu_{2} & -2 \mu_{1} \\
0 & 0 & 0 & 0 & 0 & -2 \mu_{4} & 1-2 \mu_{3}
\end{array}\right) .
$$

We also remark that $\sum_{k=-3}^{3} \phi(k)=\sum_{k \in \mathbb{Z}} \phi(k)=\int_{\mathbb{R}} \frac{1}{2 \pi} \sum_{k \in \mathbb{Z}} e^{i k \xi} \hat{\phi}(\xi) d \xi=$ $\hat{\phi}(0)=1$. Deriving the eigenvector with 0 eigenvalue such that $\sum_{k_{=-3}}^{3} \phi(k)=1$, we find that

$$
\{\phi(0), \phi( \pm 1), \phi( \pm 2), \phi( \pm 3)\}=\left\{\frac{131}{180}, \frac{37}{240},-\frac{11}{600}, \frac{1}{3600}\right\} .
$$

Thus we obtain

$$
c_{k, \ell}(=\phi(l-k))=\left\{\begin{array}{cl}
131 / 180 & \text { if } k=\ell \\
37 / 240 & \text { if } k=\ell \pm 1 \\
-11 / 600 & \text { if } k=\ell \pm 2 \\
1 / 3600 & \text { if } k=\ell \pm 3 \\
0 & \text { otherwise }
\end{array}\right.
$$

Consequently, we get the following theorem:

Theorem 2.1. For $\varphi_{2}^{D}(x)$ defined by (2.1) with $\Phi=\Phi_{2}^{D}$ we have

$$
c_{k, \ell}=\left\{\begin{array}{cl}
131 / 180 & \text { if } k=\ell, \\
37 / 240 & \text { if } k=\ell \pm 1, \\
-11 / 600 & \text { if } k=\ell \pm 2, \\
1 / 3600 & \text { if } k=\ell \pm 3 . \\
0 & \text { otherwise, }
\end{array} \text { and } \quad a_{k, \ell}=\left\{\begin{array}{cl}
-2 & \text { if } k=\ell, \\
1 & \text { if } k=\ell \pm 1 \\
0 & \text { otherwise. }
\end{array}\right.\right.
$$

Moreover, it holds that for $v \in C_{0}^{4}$

$$
\begin{aligned}
& \sum_{\nu \in \mathbb{Z}} c_{k, k+\nu} v(x+\nu h)=v(x)+O\left(h^{2}\right) \\
& \sum_{\nu=-1}^{1} a_{k, k+\nu} v(x+\nu h)=h^{2} \frac{d^{2}}{d x^{2}} v(x)+O\left(h^{4}\right) .
\end{aligned}
$$




\subsection{5-point formula for 2 nd order derivative}

With small changes of the approach in $\S 1.2$ we can also consider the 5-point formula for 2 nd order derivative. For this purpose, we replace (1.2) by

$$
\mathcal{F}^{-1}\left[|\xi \hat{\varphi}(\xi)|^{2}\right](\ell-k)=\left\{\begin{array}{cl}
\frac{5}{2} & \text { if } k=\ell, \\
-\frac{4}{3} & \text { if } k=\ell \pm 1 \\
\frac{1}{12} & \text { if } k=\ell \pm 2 \\
0 & \text { otherwise, }
\end{array}\right.
$$

which is equivalent to

$$
\begin{aligned}
& \sum_{q \in \mathbb{Z}}|(\xi+2 q \pi) \hat{\varphi}(\xi+2 q \pi)|^{2} \\
\equiv & \frac{1}{12} e^{2 i \xi}-\frac{4}{3} e^{i \xi}+\frac{5}{2} e^{i 0 \xi}-\frac{4}{3} e^{-i \xi}+\frac{1}{12} e^{-2 i \xi}\left(=\frac{4}{3} \sin ^{2} \frac{\xi}{2}\left(\sin ^{2} \frac{\xi}{2}+3\right)\right)
\end{aligned}
$$

for almost everywhere $\xi \in \mathbb{R}$. We shall define $\Phi(x)$ by

$$
\hat{\Phi}(\xi)=\frac{\hat{\varphi}(\xi)}{\left(\gamma^{-}+\gamma^{+} e^{-i \xi}\right) \hat{N}_{1}(\xi)},
$$

where $\gamma^{ \pm}=\frac{1}{2} \pm \frac{1}{\sqrt{3}}$. Then we also get

here we used

$$
\begin{aligned}
\sum_{q \in \mathbb{Z}}|\hat{\Phi}(\xi+2 q \pi)|^{2} & =\sum_{q \in \mathbb{Z}} \frac{|(\xi+2 q \pi) \hat{\varphi}(\xi+2 q \pi)|^{2}}{4 \sin ^{2} \frac{\xi+2 q \pi}{2}\left|\gamma^{-}+\gamma^{+} e^{-i(\xi+2 q \pi)}\right|^{2}} \\
& =\frac{\sum_{p \in \mathbb{Z}}|(\xi+2 q \pi) \hat{\varphi}(\xi+2 q \pi)|^{2}}{\frac{1}{12} e^{2 i \xi}-\frac{4}{3} e^{i \xi}+\frac{5}{2} e^{i 0 \xi}-\frac{4}{3} e^{-i \xi}+\frac{1}{12} e^{-2 i \xi}}
\end{aligned}
$$

$$
\begin{aligned}
& 4 \sin ^{2} \frac{\xi+2 q \pi}{2}\left|\gamma^{-}+\gamma^{+} e^{-i(\xi+2 q \pi)}\right|^{2} \\
= & 4 \sin ^{2} \frac{\xi}{2}\left|\gamma^{-}+\gamma^{+} e^{-i \xi}\right|^{2}=\frac{4}{3} \sin ^{2} \frac{\xi}{2}\left(\sin ^{2} \frac{\xi}{2}+3\right) \\
= & \frac{1}{12} e^{2 i \xi}-\frac{4}{3} e^{i \xi}+\frac{5}{2} e^{i 0 \xi}-\frac{4}{3} e^{-i \xi}+\frac{1}{12} e^{-2 i \xi} .
\end{aligned}
$$

Hence, the identity (1.4) still holds. Thus, the definition (2.3) yields

$$
\tilde{\varphi}(x)=\left\{\gamma^{-} N_{1}(\cdot)+\gamma^{+} N_{1}(\cdot-1)\right\} * \Phi(x) .
$$

Let us put $\tilde{c}_{k, \ell}:=\left\langle\tilde{\varphi}_{0, k}, \tilde{\varphi}_{0, \ell}\right\rangle_{L^{2}}$ and $\tilde{a}_{k, \ell}:=-\left\langle\frac{d}{d x} \tilde{\varphi}_{0, k}, \frac{d}{d x} \tilde{\varphi}_{0, \ell}\right\rangle_{L^{2}}$. Similarly as $\S 1.2$, by $(2.4)$ we also find that for all $\ell \in \mathbb{Z}$

$$
\begin{aligned}
\sum_{\nu \in \mathbb{Z}} \tilde{c}_{k, k+\nu} & =\sum_{q \in \mathbb{Z}}\left|\left\{\gamma^{-} \hat{N}_{1}(2 q \pi)+\gamma^{+} e^{-2 q \pi i} \hat{N}_{1}(2 q \pi)\right\} \hat{\Phi}(2 q \pi)\right|^{2} \\
& =\left|\left(\gamma^{-}+\gamma^{+}\right) \hat{\Phi}(0)\right|^{2}=1 .
\end{aligned}
$$




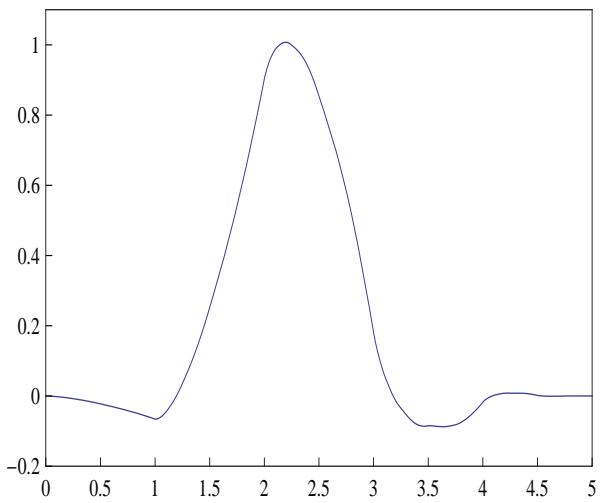

(a) $\Phi=\Phi_{2}^{D}(\operatorname{supp} \tilde{\varphi} \subset[0,5])$.

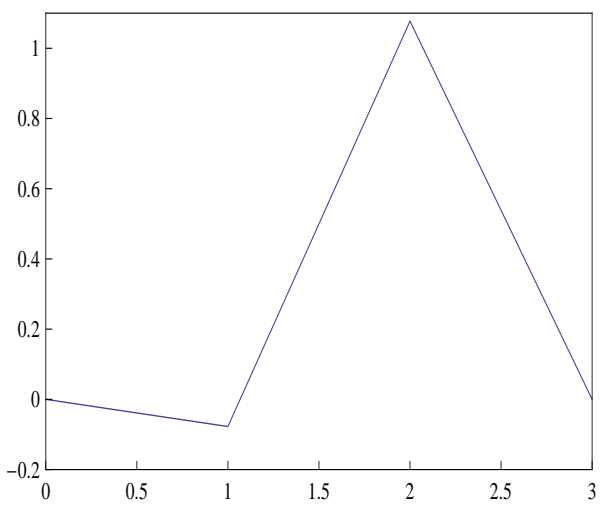

(b) $\Phi=N_{1}(\operatorname{supp} \tilde{\varphi} \subset[0,3])$.

FigURE 2. Graphs of $\tilde{\varphi}=\left\{\gamma^{-} N_{1}(x)+\gamma^{+} N_{1}(x-1)\right\} * \Phi(x)$.

We remark that $E(x)=\gamma^{+} N_{1}(x-1)+\gamma^{-} N_{1}(x)$ satisfies (i) in Definition 1.1, since $|\hat{E}(\xi)|=\left|\gamma^{-}+\gamma^{+} e^{i \xi}\right|\left|\operatorname{sinc} \frac{\xi}{2}\right|=\sqrt{\frac{1}{3} \sin ^{2} \frac{\xi}{2}}\left|\operatorname{sinc} \frac{\xi}{2}\right|$.

It remains to compute the precise value of $\tilde{c}_{k, \ell}$ for $\tilde{\varphi}(x)$. Put $\varphi=N_{1} * \Phi$ and $c_{k, \ell}=\left\langle\varphi_{0, k}, \varphi_{0, \ell}\right\rangle_{L^{2}}$ as in $\S 2.1$. Since $\tilde{\varphi}(x)=\gamma^{-} \varphi(x)+\gamma^{+} \varphi(x-1)$, we get

$$
\begin{aligned}
\tilde{c}_{k, \ell} & =\left\langle\gamma^{-} \varphi_{0, k}+\gamma^{+} \varphi_{0, k+1}, \gamma^{-} \varphi_{0, \ell}+\gamma^{+} \varphi_{0, \ell+1}\right\rangle_{L^{2}} \\
& =\left(\gamma^{+2}+\gamma^{-2}\right)\left\langle\varphi_{0, k}, \varphi_{0, \ell}\right\rangle_{L^{2}}+\gamma^{+} \gamma^{-}\left(\left\langle\varphi_{0, k}, \varphi_{0, \ell+1}\right\rangle_{L^{2}}+\left\langle\varphi_{0, k+1}, \varphi_{0, \ell}\right\rangle_{L^{2}}\right) \\
& =\frac{7}{6} c_{k, \ell}-\frac{1}{12}\left(c_{k, \ell+1}+c_{k+1, \ell}\right) .
\end{aligned}
$$


In the case of $\Phi=\Phi_{2}^{D}$, each $c_{k, \ell}$ is already given by (2.2). In the case of $\Phi=N_{1}$, since $\varphi=N_{2}$ we can easily see that

$$
c_{k, \ell}=\left\{\begin{array}{cl}
2 / 3 & \text { if } k=\ell \\
1 / 6 & \text { if } k=\ell \pm 1 \\
0 & \text { otherwise }
\end{array}\right.
$$

Consequently, we get the following theorem corresponding to Theorem 2.1:

Theorem 2.2. For $\tilde{\varphi}_{2}^{D}(x)$ defined by $(2.4)$ with $\Phi=\Phi_{2}^{D}$ (resp. $\left.N_{1}\right)$ we have

$$
\begin{gathered}
\tilde{c}_{k, \ell}=\left\{\begin{array}{cl}
3557 / 4320 & \text { if } k=\ell, \\
163 / 1350 & \text { if } k=\ell \pm 1, \\
-37 / 1080 & \text { if } k=\ell \pm 2, \\
1 / 540 & \text { if } k=\ell \pm 3, \\
-1 / 43200 & \text { if } k=\ell \pm 4, \\
0 & \text { otherwise, }
\end{array}\right. \\
\text { resp. } \tilde{c}_{k, \ell}=\left\{\begin{array}{cl}
3 / 4 & \text { if } k=\ell, \\
5 / 36 & \text { if } k=\ell \pm 1, \\
-1 / 72 & \text { if } k=\ell \pm 2, \\
0 & \text { otherwise, }
\end{array}\right)
\end{gathered}
$$

and

$$
\tilde{a}_{k, \ell}=\left\{\begin{array}{cl}
-5 / 2 & \text { if } k=\ell \\
4 / 3 & \text { if } k=\ell \pm 1 \\
-1 / 12 & \text { if } k=\ell \pm 2 \\
0 & \text { otherwise. }
\end{array}\right.
$$

Moreover, it holds that for $v \in C_{0}^{6}$

$$
\begin{aligned}
& \sum_{\nu \in \mathbb{Z}} \tilde{c}_{k, k+\nu} v(x+\nu h)=v(x)+O\left(h^{2}\right), \\
& \sum_{\nu=-2}^{2} \tilde{a}_{k, k+\nu} v(x+\nu h)=h^{2} \frac{d^{2}}{d x^{2}} v(x)+O\left(h^{6}\right) .
\end{aligned}
$$

\section{Riesz basis for higher order derivatives}

\section{1. $(2 m+1)$-point formula for $2 m$-th order derivative}

Let $N_{m}(x)$ be the B-spline of order $m(\geq 1)$ which can be defined by (see [3])

$$
\hat{N}_{m}(\xi)=\left(\frac{1-e^{-i \xi}}{i \xi}\right)^{m} \equiv\left\{\hat{N}_{1}(\xi)\right\}^{m}
$$

We shall consider the following instead of (1.3):

$$
\hat{\Phi}(\xi)=\frac{\hat{\varphi}^{(2 m)}(\xi)}{\hat{N}_{m}(\xi)} .
$$


Combining (3.1) with (1.4) we obtain $\varphi^{(2 m)}(x)$ with the elevator $\mathcal{E}(x)=N_{m}(x)$. In this section, we shall derive a further property of the elevator $N_{m}$ for applications of differential equations.

To get the $(2 m+1)$-point formula for $2 m$-th order derivative, we must find $\alpha_{\sigma}^{(r)}$ such that for any $u \in C^{2 m}$

$$
\lim _{h \rightarrow 0} \frac{\sum_{\sigma=0}^{2 m} \alpha_{\sigma}^{(2 m)} u(x+(m-\sigma) h)}{h^{2 m}}=\left(\frac{d}{d x}\right)^{2 m} u(x) .
$$

So, we expect that $\alpha_{\sigma}^{(2 m)}$,s satisfy

$$
\sum_{\sigma=0}^{2 m} \alpha_{\sigma}^{(2 m)} u(x+(m-\sigma) h)=h^{2 m}\left(\frac{d}{d x}\right)^{2 m} u(x)+O\left(h^{2 m+2}\right) .
$$

In case when $m=1$ and $r=2$, we see that $\alpha_{0}^{(2)}=1, \alpha_{1}^{(2)}=-2$ and $\alpha_{2}^{(2)}=1$ satisfy $\alpha_{0}^{(2)} u(x+h)+\alpha_{1}^{(2)} u(x)+\alpha_{2}^{(2)} u(x-h)=h^{2} \frac{d^{2}}{d x^{2}} u(x)+O\left(h^{4}\right)$. This is just the case of 3-point formula for 2 nd order derivative (see (2.2)). Using Taylor expansion, we see that cancellations occur during the odd order derivatives.

Remark 3.1. $\alpha_{\sigma}^{(2 m)}$ 's uniquely determined later from (3.2) are the best constants in sense that the remainder order is highest for the $(2 m+1)$-point formula for $2 m$-th order derivative.

Using Taylor expansion for $u(x+(m-\sigma) h)$ in $(3.2)$ and equating the coefficients of equal order derivatives on both sides of (3.2), we have

$$
\begin{aligned}
& \left(\begin{array}{cccc}
\frac{1}{0 !} & \frac{1}{0 !} & \cdots & \frac{1}{0 !} \\
\frac{(m-0) h}{1 !} & \frac{(m-1) h}{1 !} & \cdots & \frac{(m-2 m) h}{1 !} \\
\vdots & \vdots & & \vdots \\
\frac{\{(m-0) h\}^{2 m}}{(2 m) !} & \frac{\{(m-1) h\}^{2 m}}{(2 m) !} & \cdots & \frac{\{(m-2 m) h\}^{2 m}}{(2 m) !}
\end{array}\right)\left(\begin{array}{cccc}
\alpha_{0}^{(0)} & \alpha_{0}^{(1)} & \cdots & \alpha_{0}^{(2 m)} \\
\alpha_{1}^{(0)} & \alpha_{1}^{(1)} & \cdots & \alpha_{1}^{(2 m)} \\
\vdots & \vdots & & \vdots \\
\alpha_{2 m}^{(0)} & \alpha_{2 m}^{(1)} & \cdots & \alpha_{2 m}^{(2 m)}
\end{array}\right) \\
& =\left(\begin{array}{cccc}
h^{0} & 0 & \cdots & 0 \\
0 & h^{1} & \cdots & 0 \\
\vdots & \vdots & & \vdots \\
0 & 0 & \cdots & h^{2 m}
\end{array}\right) .
\end{aligned}
$$

Let us put $\lambda_{\sigma}=m-\sigma$ and $K=\left\{\alpha_{\sigma}^{(r)}\right\}_{0 \leq \sigma, r \leq 2 m}$. Then we have

$$
\left(\begin{array}{cccc}
\frac{h^{0}}{0 !} & 0 & \cdots & 0 \\
0 & \frac{h^{1}}{1 !} & \cdots & 0 \\
\vdots & \vdots & & \vdots \\
0 & 0 & \cdots & \frac{h^{2 m}}{m !}
\end{array}\right)\left(\begin{array}{cccc}
1 & 1 & \cdots & 1 \\
\lambda_{0} & \lambda_{1} & \cdots & \lambda_{2 m} \\
\vdots & \vdots & & \vdots \\
\lambda_{0}^{2 m} & \lambda_{1}^{2 m} & \cdots & \lambda_{2 m}^{2 m}
\end{array}\right) K=\left(\begin{array}{cccc}
h^{0} & 0 & \cdots & 0 \\
0 & h^{1} & \cdots & 0 \\
\vdots & \vdots & & \vdots \\
0 & 0 & \cdots & h^{2 m}
\end{array}\right) .
$$


Hence, we can solve

$$
K=\left(\begin{array}{cccc}
1 & 1 & \cdots & 1 \\
\lambda_{0} & \lambda_{1} & \cdots & \lambda_{2 m} \\
\vdots & \vdots & & \vdots \\
\lambda_{0}^{2 m} & \lambda_{1}^{2 m} & \cdots & \lambda_{2 m}^{2 m}
\end{array}\right)^{-1}\left(\begin{array}{cccc}
0 ! & 0 & \cdots & 0 \\
0 & 1 ! & \cdots & 0 \\
\vdots & \vdots & & \vdots \\
0 & 0 & \cdots & (2 m) !
\end{array}\right)
$$

Our concern in this section is $(2 m+1)$-point formula for the highest even $2 m$-th order derivatives. Therefore, we need to know the $(2 m+1)$-th and $2 m$-th columns of the inverse matrix of the Vandermonde-type and ones of $K$. From the formula of the inverse matrix of the Vandermonde-type in [4] we obtain

$$
\alpha_{\sigma}^{(2 m)}=\frac{(2 m) !}{\prod_{\ell=0, \ell \neq \sigma}^{2 m}\left(\lambda_{\ell}-\lambda_{\sigma}\right)}-\frac{(2 m-1) ! \sum_{\ell=0, \ell \neq \sigma}^{2 m} \lambda_{\ell}}{\prod_{\ell=0, \ell \neq \sigma}^{2 m}\left(\lambda_{\ell}-\lambda_{\sigma}\right)}
$$

for $\sigma=0,1, \ldots, 2 m$. We note that

$$
\sum_{\ell=0, \ell \neq \sigma}^{2 m} \lambda_{\ell}=\sum_{\ell=0}^{2 m} \lambda_{\ell}-\lambda_{\sigma}=-\lambda_{\sigma}=\sigma-m
$$

and

$$
\begin{aligned}
\prod_{\ell=0, \ell \neq \sigma}^{2 m}\left(\lambda_{\ell}-\lambda_{\sigma}\right) & =\prod_{\ell=0}^{\sigma-1}\left(\lambda_{\ell}-\lambda_{\sigma}\right) \prod_{\ell=\sigma+1}^{2 m}\left(\lambda_{\ell}-\lambda_{\sigma}\right) \\
& =(-1)^{2 m-(\sigma+1)+1} \prod_{\ell=0}^{\sigma-1}(\sigma-\ell) \prod_{\ell=\sigma+1}^{2 m}(\ell-\sigma) \\
& =(-1)^{-\sigma} \sigma !(2 m-\sigma) .
\end{aligned}
$$

Hence, with the binomial coefficient $2{ }_{2} C_{\sigma}$ we can write

$$
\alpha_{\sigma}^{(2 m)}=(-1)^{-\sigma}{ }_{2 m} C_{\sigma}(-1)^{-\sigma}{ }_{2 m} C_{\sigma} \frac{m-\sigma}{2 m}
$$

for $\sigma=0,1, \ldots, 2 m$. Here we remark that $\alpha_{\sigma}^{(2 m)}=\alpha_{2 m-\sigma}^{(2 m)}$ and that $\alpha_{\sigma}^{(2 m-1)}=$ $-\alpha_{2 m-\sigma}^{(2 m-1)}$ which means $\alpha_{m}^{(2 m-1)}=0$.

Similarly as $\S 1.2, \varphi$ must satisfies the condition

$$
\mathcal{F}^{-1}\left[\left|\xi^{m} \hat{\varphi}^{(2 m)}(\xi)\right|^{2}\right](\ell-k)=\left\{\begin{array}{cl}
(-1)^{m} \alpha_{m}^{(2 m)} & \text { if } k=\ell, \\
(-1)^{m} \alpha_{m-1}^{(2 m)} & \text { if } k=\ell \pm 1, \\
\vdots & \\
(-1)^{m} \alpha_{m-m}^{(2 m)} & \text { if } k=\ell \pm m, \\
0 & \text { otherwise, }
\end{array}\right.
$$

which is equivalent to

$$
\sum_{q \in \mathbb{Z}}\left|(\xi+2 q \pi)^{m} \hat{\varphi}^{(2 m)}(\xi+2 q \pi)\right|^{2} \equiv(-1)^{m} \sum_{\sigma=0}^{2 m} \alpha_{\sigma}^{(2 m)} e^{(m-\sigma) i \xi}\left(=4^{m} \sin ^{2 m} \frac{\xi}{2}\right)
$$


for almost everywhere $\xi \in \mathbb{R}$, here we used by (3.3)

$$
\begin{aligned}
(-1)^{m} \sum_{\sigma=0}^{2 m} \alpha_{\sigma}^{(2 m)} e^{(m-\sigma) i \xi} & =\sum_{j=0}^{2 m} 2 m C_{j}\left\{-e^{i \xi}\right\}^{m-\sigma} \\
& =\left(-e^{-i \xi}\right)^{m}\left(1-e^{i \xi}\right)^{2 m}=4^{m} \sin ^{2 m} \frac{\xi}{2}
\end{aligned}
$$

Hence, the identity (1.4) still holds for $\Phi(x)$. Thus, the definition (3.1) yields

$$
\varphi^{(2 m)}(x)=N_{m} * \Phi(x) .
$$

We remark that $\mathcal{E}(x)=N_{m}(x)$ satisfies (i) in Definition 1.1. As for (ii), we get the following theorem:

Theorem 3.1. Let us put

$a_{k, \ell}^{(2 m)}:=(-1)^{m}\left\langle\left(\frac{d}{d x}\right)^{m} \varphi_{0, k}^{(2 m)},\left(\frac{d}{d x}\right)^{m} \varphi_{0, \ell}^{(2 m)}\right\rangle_{L^{2}}\left(=\left\langle\left(\frac{d}{d x}\right)^{2 m} \varphi_{0, k}^{(2 m)}, \varphi_{0, \ell}^{(2 m)}\right\rangle_{L^{2}}\right)$

and

$a_{k, \ell}^{(2 m, r)}:=(-1)^{\left[\frac{r}{2}\right]}\left\langle\left(\frac{d}{d x}\right)^{\left[\frac{r+1}{2}\right]} \varphi_{0, k}^{(2 m)},\left(\frac{d}{d x}\right)^{\left[\frac{r}{2}\right]} \varphi_{0, k}^{(2 m)}\right\rangle_{L^{2}}=\left\langle\left(\frac{d}{d x}\right)^{r} \varphi_{0, k}^{(2 m)}, \varphi_{0, \ell}^{(2 m)}\right\rangle_{L^{2}}$

for $0 \leq r \leq 2 m-1$. Then, for $\varphi^{(2 m)}$ defined by (3.4) we have

$$
a_{k, \ell}^{(2 m)}=\left\{\begin{array}{cl}
(-1)^{m}{ }_{2 m} C_{m} & \text { if } k=\ell, \\
(-1)^{m-1}{ }_{2 m} C_{m-1} & \text { if } k=\ell \pm 1, \\
\vdots & \\
(-1)^{m-m}{ }_{2 m} C_{m-m} & \text { if } k=\ell \pm m, \\
0 & \text { otherwise. }
\end{array}\right.
$$

Moreover, it holds that for $v \in C_{0}^{2 m+2}$

$$
\begin{gathered}
\sum_{\nu \in \mathbb{Z}} a_{k, k+\nu}^{(2 m, r)} v(x+\nu h)=h^{r} \frac{d^{r}}{d x^{r}} v(x)+O\left(h^{r+2}\right) \quad(0 \leq r \leq 2 m-1), \\
\sum_{\nu=-m}^{m} a_{k, k+\nu}^{(2 m)} v(x+\nu h)=h^{2 m} \frac{d^{2 m}}{d x^{2 m}} v(x)+O\left(h^{2 m+2}\right) .
\end{gathered}
$$

Remark 3.2. For the discretization of the $2 m$-th order derivative we need the information on at least $(2 m+1)$-points. Therefore, the stiffness matrix $\left\{a_{k, \ell}^{(2 m)}\right\}$ given by (3.5) is the most sparse (the band width is $2 m+1$ ).

Proof of Theorem 3.1. (3.5) can be obtained by (3.3). Noting that $a_{k, k+\nu}^{(2 m)}=$ $\alpha_{m-\nu}^{(2 m)}$, from the construction we immediately get (3.7). It remains to prove (3.6). By Parseval's theorem we see that

$$
a_{k, \ell}^{(2 m, r)}:=\left\langle\frac{d^{r}}{d x^{r}} \varphi_{0, k}^{(2 m)}, \varphi_{0, \ell}^{(2 m)}\right\rangle_{L^{2}}=\mathcal{F}^{-1}\left[(i \xi)^{r}\left|\hat{\varphi}^{(2 m)}(\xi)\right|^{2}\right](\ell-k)
$$




$$
=\frac{1}{2 \pi} \int_{0}^{2 \pi} e^{i(\ell-k) \xi} \sum_{q \in \mathbb{Z}}\{i(\xi+2 q \pi)\}^{r}\left|\hat{\varphi}^{(2 m)}(\xi+2 q \pi)\right|^{2} d \xi .
$$

Hence, we find that

$$
\sum_{q \in \mathbb{Z}}\{i(\xi+2 q \pi)\}^{r}\left|\hat{\varphi}^{(2 m)}(\xi+2 q \pi)\right|^{2} d \xi=\sum_{\nu \in \mathbb{Z}} a_{k, k+\nu}^{(2 m, r)} e^{i \nu \xi},
$$

here we remark that $a_{k, k+\nu}^{(2 m, r)}$ is constant for all $k \in \mathbb{Z}$. Furthermore, we have for $0 \leq r \leq 2 m-1$

$$
\begin{aligned}
& \lim _{\xi \rightarrow 0} \frac{1}{(i \xi)^{r}} \sum_{\nu \in \mathbb{Z}} a_{k, k+\nu}^{(2 m, r)} e^{i \nu \xi}=\lim _{\xi \rightarrow 0} \sum_{q \in \mathbb{Z}} \frac{\{i(\xi+2 q \pi)\}^{r}\left|\hat{\varphi}^{(2 m)}(\xi+2 q \pi)\right|^{2}}{(i \xi)^{r}} \\
= & \lim _{\xi \rightarrow 0} \sum_{q \in \mathbb{Z}, q \neq 0} \frac{\{i(\xi+2 q \pi)\}^{r}\left|\sin ^{m}(\xi / 2) \hat{\Phi}(\xi+2 q \pi)\right|^{2}}{|(\xi+2 q \pi) / 2|^{2 m}(i \xi)^{r}}+\lim _{\xi \rightarrow 0} \frac{\left|\sin ^{m}(\xi / 2) \hat{\Phi}(\xi)\right|^{2}}{|\xi / 2|^{2 m}} \\
= & \lim _{\xi \rightarrow 0} \sum_{q \in \mathbb{Z}, q \neq 0} \frac{\{i(2 q \pi)\}^{r}\left|\sin ^{m} \xi \hat{\Phi}(2 q \pi)\right|^{2}}{|q \pi|^{2 m}(i \xi)^{r}}+|\hat{\Phi}(0)|^{2}=1 .
\end{aligned}
$$

Replacing $\xi$ by $h \xi$ and multiplying the both sides by $(i \xi)^{r} \hat{u}(\xi)$, we also get the identity

$$
\lim _{h \rightarrow 0} \frac{1}{h^{r}} \sum_{\nu \in \mathbb{Z}} a_{k, k+\nu}^{(2 m, r)} e^{i \nu \xi} \hat{u}(\xi)=(i \xi)^{r} \hat{u}(\xi) .
$$

By inverse Fourier transform it follows that

$$
\lim _{h \rightarrow 0} \frac{\sum_{\nu \in \mathbb{Z}} a_{k, k+\nu}^{(2 m, r)} u(x+\nu h)}{h^{r}}=\frac{d^{r}}{d x^{r}} u(x),
$$

which means (3.6).

\section{Numerical results}

Let us introduce some examples and numerical results in this section.

\begin{tabular}{|c|c|c|c|c|c|}
\hline $\begin{array}{c}\text { Riesz base } \\
\varphi\end{array}$ & $\begin{array}{c}\text { Choice of } \\
\Phi\end{array}$ & $\begin{array}{c}\text { Elevator } \\
\mathcal{E}\end{array}$ & $\begin{array}{c}\text { Length of } \\
\text { support }\end{array}$ & $\begin{array}{c}\text { Regularity } \\
\text { in } x\end{array}$ & Remainder \\
\hline$N_{2}$ & $N_{1}$ & $N_{1}$ & 2 & $C^{1}(\operatorname{Lip})$ & $O\left(h^{2}\right)$ \\
\hline$\varphi_{2}^{D}$ & $\Phi_{2}^{D}$ & $N_{1}$ & 4 & $C^{1.5}$ & $O\left(h^{2}\right)$ \\
\hline$\tilde{N}_{2}$ & $N_{1}$ & $\begin{array}{c}\gamma^{+} N_{1}(x-1) \\
+\gamma^{-} N_{1}(x)\end{array}$ & 3 & $C^{2}$ & $O\left(h^{4}\right)$ \\
\hline$\tilde{\varphi}_{2}^{D}$ & $\Phi_{2}^{D}$ & $\begin{array}{c}\gamma^{+} N_{1}(x-1) \\
+\gamma^{-} N_{1}(x)\end{array}$ & 5 & $C^{1.5}$ & $O\left(h^{4}\right)$ \\
\hline$N_{3}$ & $N_{1}$ & $N_{2}$ & 3 & $C^{2}$ & $O\left(h^{2}\right)$ \\
\hline$\stackrel{\varphi}{2}_{2}^{D}$ & $\Phi_{2}^{D}$ & $N_{2}$ & 5 & $C^{2.5}$ & $O\left(h^{2}\right)$ \\
\hline
\end{tabular}


The boundary valued problem for

$$
-\varepsilon^{2} \frac{d^{2}}{d x^{2}} u^{(\varepsilon)}+u^{(\varepsilon)}=f, \quad 0<x<1, \quad u^{(\varepsilon)}(0)=u^{(\varepsilon)}(1)=0,
$$

has a solution represented by

$$
\text { (4.1) } u^{(\varepsilon)}(x)=-\frac{\sinh (x / \varepsilon)}{\varepsilon \sinh (1 / \varepsilon)} \int_{0}^{1} \sinh \frac{y-1}{\varepsilon} f(y) d y+\frac{1}{\varepsilon} \int_{0}^{x} \sinh \frac{y-x}{\varepsilon} f(y) d y .
$$

For $f(x)=\sin 10 \pi x$, by (4.1) the exact solution is $u^{(\varepsilon)}(x)=\frac{\sin 10 \pi x}{1+100 \varepsilon^{2} \pi^{2}}$ and the errors with the Riesz bases $N_{2}$ and $\varphi_{2}^{D}$ are given by the following:

(i) The case of $\varepsilon=1$.

(ii) The case of $\varepsilon=10^{-6}$.

\begin{tabular}{|c|c|c|c|c|}
\hline Mesh size $2^{-j}$ & $E_{j}^{N_{2}}$ & $Q^{N_{2}}$ & $E_{j}^{\varphi_{2}^{D}}$ & $Q^{\varphi_{2}^{D}}$ \\
\hline$j=6$ & $1.57 \times 10^{-4}$ & 2.67 & $2.02 \times 10^{-2}$ & 6.07 \\
$j=7$ & $5.67 \times 10^{-5}$ & 2.77 & $3.58 \times 10^{-3}$ & 5.64 \\
$j=8$ & $2.02 \times 10^{-5}$ & 2.81 & $8.47 \times 10^{-4}$ & 4.23 \\
\hline
\end{tabular}

\begin{tabular}{|c|c|c|c|c|}
\hline Mesh size $2^{-j}$ & $E_{j}^{N_{2}}$ & $Q^{N_{2}}$ & $E_{j}^{\varphi_{2}^{D}}$ & $Q^{\varphi_{2}^{D}}$ \\
\hline$j=6$ & $1.58 \times 10^{-1}$ & 2.82 & $6.21 \times 10^{-4}$ & 5.57 \\
$j=7$ & $5.62 \times 10^{-2}$ & 2.81 & $1.17 \times 10^{-4}$ & 5.32 \\
$j=8$ & $2.00 \times 10^{-2}$ & 2.82 & $6.21 \times 10^{-4}$ & 5.57 \\
\hline
\end{tabular}

For $f(x)=-\varepsilon^{2}\left(9 N_{1}(3 x)-18 N_{1}(3 x-1)+N_{1}(3 x-2)\right)+N_{3}(3 x)$, the exact solution $u(x)=N_{3}(3 x)$ belongs to $H^{2}$, since $\int_{\mathbb{R}}\langle\xi\rangle^{4}|\hat{u}(\xi)|^{2} d \xi=\int_{\mathbb{R}}\langle\xi\rangle^{4}\left(\frac{\sin \xi / 6}{\xi / 6}\right)^{6} d \xi$ $<\infty$. The errors are given by the following:

(iii) The case of $\varepsilon=1$.

\begin{tabular}{|c|c|c|c|c|}
\hline Mesh size $2^{-j}$ & $E_{j}^{N_{2}}$ & $Q^{N_{2}}$ & $E_{j}^{\varphi_{2}^{D}}$ & $Q^{\varphi_{2}^{D}}$ \\
\hline$j=6$ & $1.43 \times 10^{-4}$ & 2.81 & $6.08 \times 10^{-4}$ & 4.00 \\
$j=7$ & $5.20 \times 10^{-5}$ & 2.75 & $1.52 \times 10^{-4}$ & 4.00 \\
$j=8$ & $1.84 \times 10^{-5}$ & 2.82 & $3.82 \times 10^{-5}$ & 3.98 \\
\hline
\end{tabular}

(iv) The case of $\varepsilon=10^{-6}$.

\begin{tabular}{|c|c|c|c|c|}
\hline Mesh size $2^{-j}$ & $E_{j}^{N_{2}}$ & $Q^{N_{2}}$ & $E_{j}^{\varphi_{2}^{D}}$ & $Q^{\varphi_{2}^{D}}$ \\
\hline$j=6$ & $4.68 \times 10^{-3}$ & 2.79 & $5.59 \times 10^{-4}$ & 4.00 \\
$j=7$ & $1.69 \times 10^{-3}$ & 2.77 & $1.40 \times 10^{-4}$ & 3.99 \\
$j=8$ & $6.00 \times 10^{-4}$ & 2.82 & $3.51 \times 10^{-5}$ & 3.99 \\
\hline
\end{tabular}

Here $E_{j}^{\varphi}$ is relative $L^{2}$-error between the exact solution $u^{(\varepsilon)}(x)$ and the approximation $\tilde{u}^{(\varepsilon)}(x)=\sum_{\ell=1}^{N} u_{\ell} \varphi_{j, \ell}(x)$ on $[0,1]$ defined by

$$
E_{j}^{\varphi}=E_{j}^{\varphi}(\varepsilon)=\frac{1}{\left\|u^{(\varepsilon)}\right\|_{L^{2}}} \sqrt{\sum_{\ell=0}^{2^{j}}\left\{u^{(\varepsilon)}\left(\frac{\ell}{2^{j}}\right)-\tilde{u}^{(\varepsilon)}\left(\frac{\ell}{2^{j}}\right)\right\}^{2}}
$$

and the ratio $Q^{\varphi}$ is defined by $Q^{\varphi}=E_{j-1}^{\varphi} / E_{j}^{\varphi}$. 
Now, we also consider the beam equation (4th order differential equation)

$$
\frac{d^{4}}{d x^{4}} u+u=f, \quad 0<x<1, \quad u(0)=u(1)=u^{\prime}(0)=u^{\prime}(1)=0,
$$

and its bilinear form

$$
\left\langle\frac{d^{2}}{d x^{2}} u, \frac{d^{2}}{d x^{2}} \varphi\right\rangle_{L^{2}}+\langle u, \varphi\rangle_{L^{2}}=\langle f, \varphi\rangle_{L^{2}}
$$

To derive the approximate solution we solve the following equation:

$$
\left[\left\{a_{k, \ell} h^{-3}\right\}_{1 \leq k, \ell \leq N}+\left\{c_{k, \ell} h\right\}_{1 \leq k, \ell \leq N}\right]{ }^{t}\left\{u_{\ell}\right\}_{1 \leq \ell \leq N}={ }^{t}\left\{\left\langle f, \varphi_{j, \ell}\right\rangle_{L^{2}}\right\}_{1 \leq \ell \leq N},
$$

where $c_{k, \ell}=\left\langle\varphi_{0, k}, \varphi_{0, \ell}\right\rangle$. If $\varphi$ satisfies (3.1), from (3.5) it holds that

$$
a_{k, \ell}=\left\{\begin{array}{cl}
6 & \text { if } k=\ell \\
-4 & \text { if } k=\ell \pm 1 \\
1 & \text { if } k=\ell \pm 2 \\
0 & \text { otherwise }
\end{array}\right.
$$

\begin{tabular}{|c|c|c|c|c|}
\hline Mesh size $2^{-j}$ & $E_{j}^{N_{3}}$ & $Q^{N_{3}}$ & $E_{j}^{\stackrel{\ell}{\varphi}_{2}^{D}}$ & $\tilde{Q}^{\dot{\varphi}_{2}^{D}}$ \\
\hline$j=6$ & $5.02 \times 10^{-2}$ & 2.86 & 2.44 & 3.03 \\
\hline$j=7$ & $1.77 \times 10^{-2}$ & 2.84 & $3.11 \times 10^{-1}$ & 7.86 \\
\hline$j=8$ & $6.25 \times 10^{-3}$ & 2.83 & $3.39 \times 10^{-1}$ & 0.918 \\
\hline
\end{tabular}

For $N_{3}=N_{2} * N_{1}$ and $\stackrel{\circ}{\varphi_{2}^{D}}=N_{2} * \Phi_{2}^{D}$, we get the following numerical results: (v) The case of $f(x)=\sin 10 \pi x$ and

$$
u(x)=\frac{10 \sqrt{2} \pi\left(e^{\frac{1}{\sqrt{2}}}\left(1-e^{\sqrt{2} x}\right) \sin \frac{x-1}{\sqrt{2}}-\left(e^{\sqrt{2}}-e^{\sqrt{2} x}\right) \sin \frac{x}{\sqrt{2}}\right)+e^{\frac{x}{\sqrt{2}}}\left(e^{\sqrt{2}}-1-2 e^{\frac{1}{\sqrt{2}}} \sin \frac{1}{\sqrt{2}}\right) \sin 10 \pi x}{\left(1+10000 \pi^{4}\right) e^{\frac{x}{\sqrt{2}}}\left(e^{\sqrt{2}}-1-2 e^{\frac{1}{\sqrt{2}}} \sin \frac{1}{\sqrt{2}}\right)} .
$$

(vi) The case of $f(x)=x$ and

$$
\begin{aligned}
u(x)= & \left\{\left(e^{\frac{2 x+3}{\sqrt{2}}}+e^{\frac{1}{\sqrt{2}}}\right)\left(\sqrt{2} e^{\sqrt{2}}-\sqrt{2}+1\right)-2 e^{\frac{1}{\sqrt{2}}}\right) \sin \frac{x-1}{\sqrt{2}} \\
& -e^{\frac{1}{\sqrt{2}}}\left(e^{\sqrt{2} x}\left(e^{\sqrt{2}}-2-\sqrt{2}\right)-e^{\sqrt{2}}(2-\sqrt{2})+1\right) \cos \frac{x-1}{\sqrt{2}} \\
& +\sqrt{2}\left(e^{\sqrt{2}}-1\right)\left(e^{\sqrt{2} x}-e^{\sqrt{2}}\right) \sin \frac{x}{\sqrt{2}}+\left(e^{\frac{3}{\sqrt{2}}}-e^{\frac{2 x+1}{\sqrt{2}}}\right) \sin \frac{x+1}{\sqrt{2}} \\
& +\sqrt{2} e^{\sqrt{2}}\left(e^{\sqrt{2} x}-1\right)\left(\cos \frac{x}{\sqrt{2}}-\cos \frac{x-2}{\sqrt{2}}\right) \\
& \left.+\left((\sqrt{2}-1) e^{\frac{3}{\sqrt{2}}}-(1+\sqrt{2}) e^{\frac{2 x+1}{\sqrt{2}}}\right) \cos \frac{x+1}{\sqrt{2}}\right\} \\
& \times\left\{2 e^{\frac{x+2}{\sqrt{2}}}(-2+\cos \sqrt{2}+\cosh \sqrt{2})\right\}^{-1} \\
& +x .
\end{aligned}
$$




\begin{tabular}{|c|c|c|c|c|}
\hline Mesh size $2^{-j}$ & $E_{j}^{N_{3}}$ & $Q^{N_{3}}$ & $E_{j}^{\stackrel{\circ}{2}_{2}^{D}}$ & $\tilde{Q}^{\stackrel{\circ}{\varphi}_{2}^{D}}$ \\
\hline$j=6$ & $9.02 \times 10^{-3}$ & 2.83 & 1.33 & 1.33 \\
$j=7$ & $3.19 \times 10^{-3}$ & 2.83 & $9.71 \times 10^{-1}$ & 1.37 \\
$j=8$ & $1.13 \times 10^{-3}$ & 2.83 & $6.97 \times 10^{-1}$ & 1.39 \\
\hline
\end{tabular}

(vii) The case of $f(x)=N_{5}(5 x)+625 N_{1}(5 x)-2500 N_{1}(5 x-1)+3750 N_{1}(5 x-$ $2)-2500 N_{1}(5 x-3)+625 N_{1}(5 x-4)$ and $u(x)=N_{5}(5 x) \in H^{4}$.

\begin{tabular}{|c|c|c|c|c|}
\hline Mesh size $2^{-j}$ & $E_{j}^{N_{3}}$ & $Q^{N_{3}}$ & $E_{j}^{\stackrel{\circ}{L}^{D}}$ & $\tilde{Q}^{\stackrel{\circ}{\varphi}_{2}^{D}}$ \\
\hline$j=6$ & $7.81 \times 10^{-3}$ & 2.83 & $1.14 \times 10^{-4}$ & 5.18 \\
$j=7$ & $2.76 \times 10^{-3}$ & 2.83 & $1.14 \times 10^{-4}$ & 5.18 \\
$j=8$ & $9.75 \times 10^{-4}$ & 2.83 & $2.20 \times 10^{-4}$ & 5.17 \\
\hline
\end{tabular}

(viii) The case of $f(x)=N_{4}(4 x)-256 \delta(x-1 / 4)+384 \delta(x-1 / 2)-256 \delta(x-3 / 4)$ and $u(x)=N_{4}(4 x) \in H^{s}$ for $s<3.5(<4)$.

\begin{tabular}{|c|c|c|c|c|}
\hline Mesh size $2^{-j}$ & $E_{j}^{N_{3}}$ & $Q^{N_{3}}$ & $E_{j}^{\stackrel{\circ}{2}^{D}}$ & $\tilde{Q}^{\stackrel{\leftrightarrow}{\varphi}_{2}}$ \\
\hline$j=6$ & $6.15 \times 10^{-3}$ & 2.85 & $5.98 \times 10^{-2}$ & 2.87 \\
$j=7$ & $2.17 \times 10^{-3}$ & 2.83 & $2.10 \times 10^{-2}$ & 2.85 \\
$j=8$ & $7.67 \times 10^{-4}$ & 2.83 & $7.41 \times 10^{-3}$ & 2.83 \\
\hline
\end{tabular}

\section{Concluding remarks}

The method with the elevated Riesz bases converts a continuous operator to a discrete problem by featuring the highest order derivatives. Therefore, we can consider the following advantages:

(1) For $-\varepsilon^{2} \frac{d^{2}}{d x^{2}} u^{(\varepsilon)}+u^{(\varepsilon)}=f$, if $\varepsilon>0$ is smaller, $-\varepsilon^{2} \frac{d^{2}}{d x^{2}}$ gives more perturbation to the solution. The influence of the parameter $\varepsilon>0$ can be reduced to some extent. Actually, for $\varphi_{2}^{D}$ the relative $L^{2}$-error $E_{j}^{\varphi_{2}^{D}}$ is stable for a smaller $\varepsilon>0$ (see $E_{j}^{\varphi_{2}^{D}}$ in (i), (ii), (iii), (iv)).

(2) For $(-1)^{m} \frac{d^{2 m}}{d x^{2 m}} u+u=f$, if the solution has lower regularity, $(-1)^{m} \frac{d^{2 m}}{d x^{2 m}}$ plays a more role in the structure of the equation. When we consider the solution in $H^{2 m}$ or of less regularity in $H^{s}(s<2 m)$, for instance $m=2$, the relative $L^{2}$-errors $E_{j}^{N_{3}}$ and $E_{j}^{\grave{\varphi}_{2}^{D}}$ keep good results even in comparison with $m=1$ (see (iii), (vii), (viii)).

Acknowledgements. The authors thank the referee for deep and constructive suggestions which improved the paper considerably.

\section{References}

[1] G. Beylkin, On the representation of operators in bases of compactly supported wavelets, SIAM J. Numer. Anal. 29 (1992), no. 6, 1716-1740.

[2] C. K. Chui, An Introduction to Wavelets, Wavelet Analysis and its Applications, 1, Academic Press, Boston, MA, 1992. [3] $\frac{}{1997 .}$, Wavelets: A Mathematical Tool for Signal Processing, SIAM, Philadelphia, PA, 
[4] F. Colombini and T. Kinoshita, On the Gevrey well posedness of the Cauchy problem for weakly hyperbolic equations of higher order, J. Differential Equations 186 (2002), no. 2, 394-419.

[5] I. Daubechies, Ten Lectures on Wavelets, CBMS-NSF Regional Conference Series in Applied Mathematics, 61, SIAM, Philadelphia, PA, 1992.

[6] M. Ersoy, A simple and efficient new algorithm to increase the regularity and vanishing moments of biorthogonal wavelets, preprint.

[7] N. Fukuda and T. Kinoshita, On non-symmetric orthogonal spline wavelets, Southeast Asian Bulletin of Mathematics, to appear.

[8] _ On the new family of wavelets interpolating to the Shannon wavelet, JSIAM Lett. 3 (2011), 33-36.

[9] - On the construction of new families of wavelets, Jpn. J. Ind. Appl. Math. 29 (2012), no. 1, 63-82.

[10] R. H. Gallagher, Finite Element Analysis, Prentice-Hall, Inc., Englewood Cliffs, New Jersey, 1975.

[11] E. Hernández and G. Weiss, A First Course on Wavelets, CRC Press, Boca Raton, Florida, 1996

[12] A. K. Louis, P. Maass, and A. Rieder, Wavelets: theory and applications, Wiley, Chichester, 1998.

[13] H. C. Martin and G. F. Carey, Introduction to Finite Element Analysis, McGraw-Hill Book Co., New York-Düsseldorf-Johannesburg, 1973.

[14] J. O. Strömberg, A modified Franklin system and higher-order spline systems on $\mathbb{R}^{n}$ as unconditional bases for Hardy spaces, Conference on harmonic analysis in honor of Antoni Zygmund, Vol. I, II (Chicago, Ill., 1981), 475-494, Wadsworth Math. Ser., Wadsworth, Belmont, CA, 1983.

[15] G. Strang and G. J. Fix, An Analysis of the Finite Element Method, Prentice-Hall Inc., Englewood Cliffs, New Jersey, 1973.

[16] W. C. Shann, J. Tzeng, and S. W. Chen, The leveraged wavelets and Galerkin-wavelets methods, preprint.

[17] J. C. Xu and W. C. Shann, Galerkin-wavelet methods for two-point boundary value problems, Numer. Math. 63 (1992), no. 1, 123-144.

\author{
NAOHIRO FUKUDA \\ INSTITUTE OF MATHEMATICS \\ UNIVERSITY OF TSUKUBA \\ TSUKUBA, 305-8571, JAPAN \\ E-mail address: naohiro-f@math.tsukuba.ac.jp \\ TAMOTU Kinoshita \\ INSTITUTE OF MATHEMATICS \\ UNIVERSITY OF TSUKUBA \\ TSUKUBA, 305-8571, JAPAN \\ E-mail address: kinosita@math.tsukuba.ac.jp \\ TAKAYUKI KuBO \\ INSTITUTE OF MATHEMATICS \\ UNIVERSITY OF TSUKUBA \\ TSUKUBA, 305-8571, JAPAN \\ E-mail address: tkubo@math.tsukuba.ac.jp
}

